\title{
Robust Equilibria of Potential Games
}

\author{
BY TAKASHI UI \\ Institute of Policy and Planning Sciences \\ University of Tsukuba \\ 1-1-1 Tennodai, Tsukuba, Ibaraki 305-8573, Japan \\ E-mail: oui@shako.sk.tsukuba.ac.jp
}

July 1998

Revised, October 2000

\begin{abstract}
Potential games are games with potential functions. Technically, the potential function defines a refinement concept. We provide justification for this refinement concept using the notion of robustness of equilibria. A Nash equilibrium of a complete information game is said to be robust if every incomplete information game where payoffs are almost always given by the complete information game has an equilibrium which generates behavior close to the Nash equilibrium. We show that Nash equilibria that maximize potential functions are generically robust.
\end{abstract}

Keywords: Incomplete information, refinements, robustness, potential games. 


\title{
Robust Equilibria of Potential Games
}

\author{
BY TAKASHI UI ${ }^{1}$
}

\section{INTRODUCTION}

Potential Games, as considered by Monderer and Shapley (1996), are games with potential functions. Potential functions are functions of action profiles such that the difference induced by a single deviation is equal to that of the deviator's payoff function. Monderer and Shapley (1996) demonstrated that the set of action profiles maximizing the potential function is a subset of Nash equilibria and that this subset does not depend upon a particular potential function. Monderer and Shapley (1996, p.135) wrote, "at least technically, the potential defines a refinement concept."

The observation of Monderer and Shapley (1996) drives us to the question what can justify this refinement concept. ${ }^{2}$ The purpose of this paper is to provide a possible justification, using the notion of robustness of equilibria to incomplete information considered by Kajii and Morris (1997a, 1997b). A Nash equilibrium of a complete information game is said to be robust if every incomplete information game with payoffs almost always given by the complete information game has an equilibrium which generates behavior close to the Nash equilibrium. We show that Nash equilibria that maximize potential functions are generically robust in the sense of Kajii and Morris (1997b).

The robustness of equilibria in Kajii and Morris (1997a) and that in Kajii and Morris (1997b) are slightly different. In the definition of robustness, Kajii and Morris (1997a) considered incomplete information games where all types either have payoffs given by the complete information game or have any payoffs. Kajii and Morris (1997b) restricted attention to incomplete information games where all types either 
have payoffs given by the complete information game or have a dominant strategy to choose one action. Thus, if a Nash equilibrium is robust in the sense of Kajii and Morris (1997a) then it is also robust in the sense of Kajii and Morris (1997b), though the difference between them is an open question.

Kajii and Morris (1997a, 1997b) were able to present two classes of games in which robust equilibria exist. One is a class of games with unique correlated equilibria. The other is a class of games with $\mathbf{p}$-dominant equilibria such that $\sum_{i} p_{i}<1$. Our result states that potential games are another class of games in which robust equilibria exist. To prove the result, we use the "potential function" technique, instead of the "higher order belief" technique Kajii and Morris (1997a) used.

The organization of this paper is as follows. Section 2 introduces potential games. Section 3 defines robust equilibria. Section 4 provides the main result.

\section{POTENTIAL GAMES}

A complete information game consists of a finite set of players $N=\{1, \ldots, n\}$, a finite action set $A_{i}$ for $i \in N$, and a payoff function $g_{i}: A \rightarrow \mathbb{R}$ for $i \in N$ where $A=\prod_{i \in N} A_{i}$. We write $A_{-i}=\prod_{j \neq i} A_{j}$ and $a_{-i}=\left(a_{1}, \ldots, a_{i-1}, a_{i+1}, \ldots, a_{n}\right) \in A_{-i}$. Because we will fix $N$ and $A$ throughout the paper, we simply denote a complete information game by $\mathbf{g}=\left\{g_{i}\right\}_{i \in N}$.

A potential game is a complete information game formally defined by Monderer and Shapley (1996).

DEFINITION 1 A complete information game $\mathbf{g}$ is a potential game if there exists a potential function $G: A \rightarrow \mathbb{R}$ such that

$$
g_{i}\left(a_{i}, a_{-i}\right)-g_{i}\left(a_{i}^{\prime}, a_{-i}\right)=G\left(a_{i}, a_{-i}\right)-G\left(a_{i}^{\prime}, a_{-i}\right)
$$


for all $i \in N, a_{i}, a_{i}^{\prime} \in A_{i}$, and $a_{-i} \in A_{-i}$.

Monderer and Shapley (1996) showed that a potential function is uniquely defined up to an additive constant.

LEMMA 1 Let $G$ and $G^{\prime}$ be potential functions of $\mathbf{g}$. There exists a constant $c$ such that $G(a)=G^{\prime}(a)+c$ for all $a \in A$.

By the definition, it is clear that action profiles maximizing potential functions are Nash equilibria. By the lemma, the argmax sets of potential functions do not depend upon particular potential functions. Accordingly, the argmax sets of potential functions refine equilibrium sets. For more properties and examples ${ }^{3}$ of potential games, see Monderer and Shapley (1996) and Ui (2000).

\section{ROBUST EQUILIBRIA}

Consider an incomplete information game with the set of players $N$ and the action space $A$. Let $T_{i}$ be a countable set of types of player $i \in N$. The state space consists of all type profiles, $T=\prod_{i \in N} T_{i}$. We write $T_{-i}=\prod_{j \neq i} T_{j}$ and $t_{-i}=$ $\left(t_{1}, \ldots, t_{i-1}, t_{i+1}, \ldots, t_{n}\right) \in T_{-i}$. Let $P \in \Delta(T)$ be the prior probability distribution on $T$ with $P\left(\left\{t_{i}\right\} \times T_{-i}\right)>0$ for all $i \in N$ and $t_{i} \in T_{i}$. A payoff function of player $i$ is a bounded function $u_{i}: A \times T \rightarrow \mathbb{R}$. Because we will fix $T, N$, and $A$ throughout the paper, we simply denote an incomplete information game by $(\mathbf{u}, P)$ where $\mathbf{u}=\left\{u_{i}\right\}_{i \in N}$.

A (mixed) strategy of player $i \in N$ is a function $\sigma_{i}: T_{i} \rightarrow \Delta\left(A_{i}\right)$ where $\Delta\left(A_{i}\right)$ is the set of probability distributions on $A_{i}$. We write $\Sigma_{i}$ for the set of strategies of player $i$, and write $\Sigma=\prod_{i \in N} \Sigma_{i}, \sigma=\left(\sigma_{1}, \ldots, \sigma_{n}\right) \in \Sigma, \Sigma_{-i}=\prod_{j \neq i} \Sigma_{j}$, and $\sigma_{-i}=$ $\left(\sigma_{1}, \ldots, \sigma_{i-1}, \sigma_{i+1}, \ldots, \sigma_{n}\right) \in \Sigma_{-i}$. We write $\sigma_{i}\left(a_{i} \mid t_{i}\right)$ for the probability of action $a_{i}$ 
given $\sigma_{i} \in \Sigma_{i}$ and $t_{i} \in T_{i}$. We write $\sigma(a \mid t)=\prod_{i \in N} \sigma_{i}\left(a_{i} \mid t_{i}\right)$ and $\sigma_{-i}\left(a_{-i} \mid t_{-i}\right)=$ $\prod_{j \neq i} \sigma_{j}\left(a_{j} \mid t_{j}\right)$. We write $\sigma_{P}(a)=\sum_{t \in T} P(t) \sigma(a \mid t)$.

The payoff of strategy profile $\sigma \in \Sigma$ to player $i$ is

$$
U_{i}(\sigma)=\sum_{t \in T} \sum_{a \in A} P(t) \sigma(a \mid t) u_{i}(a, t) .
$$

A strategy profile $\sigma \in \Sigma$ is a Bayesian Nash equilibrium of $(\mathbf{u}, P)$ if, for each $i \in N$,

$$
U_{i}(\sigma) \geq U_{i}\left(\sigma_{i}^{\prime}, \sigma_{-i}\right)
$$

for all $\sigma_{i}^{\prime} \in \Sigma_{i}$

Let $\mathbf{g}$ be given. We consider the following subset of $T_{i}$ :

$T_{i}^{u_{i}}=\left\{t_{i} \in T_{i} \mid u_{i}\left(a,\left(t_{i}, t_{-i}\right)\right)=g_{i}(a)\right.$ for all $a \in A, t_{-i} \in T_{-i}$ with $\left.P\left(\left(t_{i}, t_{-i}\right)\right)>0\right\}$.

When $t_{i} \in T_{i}^{u_{i}}$ is realized, payoffs of player $i$ are given by $g_{i}$, and he knows his payoffs.

We write $T^{\mathbf{u}}=\prod_{i \in N} T_{i}^{u_{i}} \cdot{ }^{4}$

DEFINITION 2 An incomplete information game $(\mathbf{u}, P)$ is an $\varepsilon$-elaboration of $\mathbf{g}$ if $P\left(T^{\mathbf{u}}\right)=1-\varepsilon$ for $\varepsilon \in[0,1]$.

Payoffs of a 0 -elaboration are given by $\mathbf{g}$ with probability 1 and every player knows his payoffs. Thus, if $a^{*} \in A$ is a Nash equilibrium of $\mathbf{g}$ then the 0 -elaboration has a Bayesian Nash equilibrium $\sigma \in \Sigma$ with $\sigma\left(a^{*} \mid t\right)=1$ for all $t \in T$, i.e., $\sigma_{P}\left(a^{*}\right)=1$. We say that $a^{*}$ is robust if, for small $\varepsilon>0$, every $\varepsilon$-elaboration of $\mathbf{g}$ has a Bayesian Nash equilibrium $\sigma \in \Sigma$ with $\sigma_{P}\left(a^{*}\right)$ close to 1 . The robustness of equilibria to all elaborations is defined by Kajii and Morris (1997a). ${ }^{5}$

DEFINITION 3 Action profile $a^{*}$ is robust to all elaborations in $\mathbf{g}$ if, for every $\delta>0$, there exists $\bar{\varepsilon}>0$ such that, for all $\varepsilon \leq \bar{\varepsilon}$, every $\varepsilon$-elaboration of $\mathbf{g}$ has a Bayesian Nash equilibrium $\sigma$ with $\sigma_{P}\left(a^{*}\right) \geq 1-\delta$. 
We call $t_{i} \in T_{i} \backslash T_{i}^{u_{i}}$ a committed type if player $i$ of type $t_{i}$ has a strictly dominant action $a_{i}^{t_{i}} \in A_{i}$ with

$$
u_{i}\left(\left(a_{i}^{t_{i}}, a_{-i}\right),\left(t_{i}, t_{-i}\right)\right)>u_{i}\left(\left(a_{i}, a_{-i}\right),\left(t_{i}, t_{-i}\right)\right)
$$

for all $a_{i} \in A_{i} \backslash\left\{a_{i}^{t_{i}}\right\}, a_{-i} \in A_{-i}$, and $t_{-i} \in T_{-i}$ with $P\left(\left(t_{i}, t_{-i}\right)\right)>0$. In this paper, we focus on the following special class of $\varepsilon$-elaborations.

DEFINITION 4 An $\varepsilon$-elaboration of $\mathbf{g}$ is said to be canonical if every $t_{i} \in T_{i} \backslash T_{i}^{u_{i}}$ is a committed type for all $i \in N$.

By considering canonical $\varepsilon$-elaborations, we can define a slightly weaker version of the robustness. The robustness of equilibria to canonical elaborations is defined by Kajii and Morris (1997b).

DEFINITION 5 Action profile $a^{*}$ is robust to canonical elaborations in $\mathbf{g}$ if, for every $\delta>0$, there exists $\bar{\varepsilon}>0$ such that, for all $\varepsilon \leq \bar{\varepsilon}$, every canonical $\varepsilon$-elaboration of $\mathbf{g}$ has a Bayesian Nash equilibrium $\sigma$ with $\sigma_{P}\left(a^{*}\right) \geq 1-\delta$.

Clearly, if a Nash equilibrium is robust to all elaborations then it is also robust to canonical elaborations. The difference between them, however, is an open question. ${ }^{6}$ For the purpose of justifying the argmax sets of potential functions as refinements, we use the robustness to canonical elaborations.

Kajii and Morris (1997a) demonstrated that some games have no robust equilibria. More precisely, there exist open sets of games with a unique (strict) Nash equilibrium which is not robust to canonical elaborations. Kajii and Morris (1997a) provided two sufficient conditions for robustness. ${ }^{7}$ One applies to games with unique correlated equilibria.

THEOREM 1 If action profile $a^{*}$ is a unique correlated equilibrium of $\mathbf{g}$ then it is robust to all elaborations. 
The other applies to games with $\mathbf{p}$-dominant equilibria such that $\sum_{i \in N} p_{i}<1$.

Definition 6 Let $\mathbf{p}=\left(p_{1}, \ldots, p_{n}\right) \in[0,1]^{n}$. Action profile $a^{*}$ is a $\mathbf{p}$-dominant equilibrium of $\mathbf{g}$ if, for all $i \in N$ and $\lambda \in \Delta\left(A_{-i}\right)$ with $\lambda\left(a_{-i}^{*}\right) \geq p_{i}$,

$$
a_{i}^{*} \in \arg \max _{a_{i} \in A_{i}} \sum_{a_{-i} \in A_{-i}} \lambda\left(a_{-i}\right) g_{i}\left(a_{i}, a_{-i}\right) .
$$

THEOREM 2 If action profile $a^{*}$ is a $\mathbf{p}$-dominant equilibrium of $\mathbf{g}$ with $\sum_{i \in N} p_{i}<1$ then it is robust to all elaborations.

The main result of this paper applies to potential games; we will show that if $a^{*}$ is a unique potential maximizer then it is robust to canonical elaborations. Before presenting the result, we demonstrate that conditions of Theorem 1 and Theorem 2 are, in general, too strong for potential games. Consider the following game.

\section{Player 2}

\begin{tabular}{cc|c|c|c|} 
& & $a_{2}$ & $b_{2}$ & $c_{2}$ \\
\hline$a_{1}$ & 4,4 & 2,2 & 0,0 \\
\hline$b_{1}$ & 0,0 & 3,3 & 0,0 \\
\hline$c_{1}$ & 2,2 & 0,0 & 3,3 \\
\hline
\end{tabular}

This is a potential game with a potential function $G=g_{1}=g_{2}$, which has three strict Nash equilibria, $\left(a_{1}, a_{2}\right),\left(b_{1}, b_{2}\right)$, and $\left(c_{1}, c_{2}\right)$. Due to the multiplicity of equilibria, Theorem 1 does not apply to this game. In addition, we can show that this game has no $\left(p_{1}, p_{2}\right)$-dominant equilibria with $p_{1}+p_{2}<1$.

Consider $\left(a_{1}, a_{2}\right)$. Let $\lambda \in \Delta\left(A_{2}\right)$ be such that $\lambda\left(a_{2}\right)<3 / 5$ and $\lambda\left(c_{2}\right)=1-\lambda\left(a_{2}\right)$, and $\mu \in \Delta\left(A_{1}\right)$ be such that $\mu\left(a_{1}\right)<3 / 5$ and $\mu\left(b_{1}\right)=1-\mu\left(a_{1}\right)$. Then $\left\{c_{1}\right\}=$ $\arg \max _{x_{1} \in A_{1}} \sum_{x_{2} \in A_{2}} \lambda\left(x_{2}\right) g_{1}\left(x_{1}, x_{2}\right)$ and $\left\{b_{2}\right\}=\arg \max _{x_{2} \in A_{2}} \sum_{x_{1} \in A_{1}} \mu\left(x_{1}\right) g_{2}\left(x_{1}, x_{2}\right)$. Thus, if $\left(a_{1}, a_{2}\right)$ is a $\left(p_{1}, p_{2}\right)$-dominant equilibrium then it must be true that $p_{1} \geq 3 / 5$ and $p_{2} \geq 3 / 5$, i.e., $p_{1}+p_{2}>1$. Similarly, we can show that if $\left(b_{1}, b_{2}\right)$ or $\left(c_{1}, c_{2}\right)$ is a $\left(p_{1}, p_{2}\right)$-dominant equilibrium then it must be true that $p_{1}+p_{2}>1$. Accordingly, Theorem 2 does not apply to this game. ${ }^{8}$ 


\section{ROBUST EQUILIBRIA OF POTENTIAL GAMES}

We introduce a potential-like function of an $\varepsilon$-elaboration of a potential game.

DEFINITION 7 Let $(\mathbf{u}, P)$ be an $\varepsilon$-elaboration of a potential game $\mathbf{g}$ with a potential function $G$. Define a function $V: \Sigma \rightarrow \mathbb{R}$ such that

$$
V(\sigma)=\sum_{t \in T} \sum_{a \in A} P(t) \sigma(a \mid t) G(a) .
$$

$V$ is called an elaboration potential of $(\mathbf{u}, P)$.

An elaboration potential $V$ is not a potential function ${ }^{9}$ of $(\mathbf{u}, P)$, but close to it in the following sense. ${ }^{10}$

LEMMA 2 Let $(\mathbf{u}, P)$ be an E-elaboration of a potential game $\mathbf{g}$ with a potential function $G$. Let $V$ be the elaboration potential. For each $i \in N$, if $\sigma_{i}, \sigma_{i}^{\prime} \in \Sigma_{i}$ is such that $\sigma_{i}\left(t_{i}\right)=\sigma_{i}^{\prime}\left(t_{i}\right)$ for all $t_{i} \in T_{i} \backslash T_{i}^{u_{i}}$ then

$$
U_{i}\left(\sigma_{i}, \sigma_{-i}\right)-U_{i}\left(\sigma_{i}^{\prime}, \sigma_{-i}\right)=V\left(\sigma_{i}, \sigma_{-i}\right)-V\left(\sigma_{i}^{\prime}, \sigma_{-i}\right)
$$

for all $\sigma_{-i} \in \Sigma_{-i}$.

Let $(\mathbf{u}, P)$ be a canonical $\varepsilon$-elaboration. Let $a_{i}^{t_{i}}$ be the strictly dominant action of a committed type $t_{i} \in T_{i} \backslash T_{i}^{u_{i}}$. We consider $\Sigma_{i}^{u_{i}} \subseteq \Sigma_{i}$ such that

$$
\Sigma_{i}^{u_{i}}=\left\{\sigma_{i} \in \Sigma_{i} \mid \sigma_{i}\left(a_{i}^{t_{i}} \mid t_{i}\right)=1 \text { for all } t_{i} \in T_{i} \backslash T_{i}^{u_{i}}\right\} .
$$

We write $\Sigma^{\mathbf{u}}=\prod_{i \in N} \Sigma_{i}^{u_{i}}$. In potential games, action profiles maximizing potential functions are Nash equilibria. A similar claim is true for canonical $\varepsilon$-elaborations of potential games. ${ }^{11}$

LEMMA 3 Let $(\mathbf{u}, P)$ be a canonical E-elaboration of a potential game $\mathbf{g}$ with a potential function $G$. Let $V$ be the elaboration potential. Then $\arg \max _{\sigma \in \Sigma^{u}} V(\sigma)$ is nonempty and $\sigma^{*} \in \arg \max _{\sigma \in \Sigma^{\mathbf{u}}} V(\sigma)$ is a Bayesian Nash equilibrium of $(\mathbf{u}, P)$. 
If $\sigma^{*} \in \arg \max _{\sigma \in \Sigma^{u}} V(\sigma)$ and $\left\{a^{*}\right\}=\arg \max _{a \in A} G(a)$, we can show that $\sigma_{P}^{*}\left(a^{*}\right)$ is close to 1 when $\varepsilon$ is close to 0 , which leads us to our main result:

THEOREM 3 Let $\mathbf{g}$ be a potential game with a potential function $G$. Suppose that $\left\{a^{*}\right\}=\arg \max _{a \in A} G(a)$. Then $a^{*}$ is robust to canonical elaborations in $\mathbf{g}$.

Proof Let $(\mathbf{u}, P)$ be a canonical $\varepsilon$-elaboration of $\mathbf{g}$. Let $V$ be the elaboration potential. Let $\sigma^{*}$ be a Bayesian Nash equilibrium of $(\mathbf{u}, P)$ such that $\sigma^{*} \in \arg \max _{\sigma \in \Sigma^{\mathbf{u}}} V(\sigma)$, which exists due to Lemma 3.

Define $G^{*}=G\left(a^{*}\right), G^{\prime}=\max _{a \in A \backslash\left\{a^{*}\right\}} G(a)$, and $G^{\prime \prime}=\min _{a \in A} G(a)$. Note that $G^{*}>G^{\prime} \geq G^{\prime \prime}$. Let $\sigma \in \Sigma^{\mathbf{u}}$ be such that $\sigma_{i}\left(a_{i}^{*} \mid t_{i}\right)=1$ for all $t_{i} \in T_{i}^{u_{i}}$ and $i \in N$.

We have

$$
\begin{aligned}
V\left(\sigma^{*}\right) \geq V(\sigma) & =\sum_{t \in T^{\mathbf{u}}} \sum_{a \in A} P(t) \sigma(a \mid t) G(a)+\sum_{t \in T \backslash T^{\mathbf{u}}} \sum_{a \in A} P(t) \sigma(a \mid t) G(a) \\
& =P\left(T^{\mathbf{u}}\right) G\left(a^{*}\right)+\sum_{t \in T \backslash T^{\mathbf{u}}} P(t) \sum_{a \in A} \sigma(a \mid t) G(a) \\
& \geq P\left(T^{\mathbf{u}}\right) G^{*}+\left[1-P\left(T^{\mathbf{u}}\right)\right] G^{\prime \prime}=(1-\varepsilon) G^{*}+\varepsilon G^{\prime \prime} .
\end{aligned}
$$

We also have

$$
\begin{aligned}
V\left(\sigma^{*}\right) & =\sum_{a \in A}\left[\sum_{t \in T} P(t) \sigma^{*}(a \mid t)\right] G(a) \\
& =\sum_{a \in A} \sigma_{P}^{*}(a) G(a) \\
& =\sigma_{P}^{*}\left(a^{*}\right) G\left(a^{*}\right)+\sum_{a \in A \backslash\left\{a^{*}\right\}} \sigma_{P}^{*}(a) G(a) \\
& \leq \sigma_{P}^{*}\left(a^{*}\right) G^{*}+\left[1-\sigma_{P}^{*}\left(a^{*}\right)\right] G^{\prime} .
\end{aligned}
$$

Combining the above inequalities, we have

$$
(1-\varepsilon) G^{*}+\varepsilon G^{\prime \prime} \leq \sigma_{P}^{*}\left(a^{*}\right) G^{*}+\left[1-\sigma_{P}^{*}\left(a^{*}\right)\right] G^{\prime}
$$

and thus

$$
\sigma_{P}^{*}\left(a^{*}\right) \geq 1-\varepsilon \frac{G^{*}-G^{\prime \prime}}{G^{*}-G^{\prime}}
$$


This implies that, for every $\delta>0$, if we choose $\bar{\varepsilon}=\delta\left(G^{*}-G^{\prime}\right) /\left(G^{*}-G^{\prime \prime}\right)>0$ then, for all $\varepsilon \leq \bar{\varepsilon}$, every canonical $\varepsilon$-elaboration of $\mathbf{g}$ has a Bayesian Nash equilibrium $\sigma^{*}$ with $\sigma_{P}^{*}\left(a^{*}\right) \geq 1-\delta$, which completes the proof. Q.E.D.

REMARK 1 Theorem 3 does not necessarily imply that $a^{*}$ is the unique robust equilibrium. On the other hand, as shown by Kajii and Morris (1997a, Corollary 5.6), a strictly $\mathbf{p}$-dominant equilibrium with $\sum_{i} p_{i}<1$ is the unique robust equilibrium. It is an open question when robust equilibria are unique, if they exist.

REMARK 2 Using the similar argument, we can show that $a^{*}$ is robust to a class of elaborations that have Bayesian potential functions in the sense of Facchini et al. (1997). This robustness is considered to be stronger than the robustness to canonical elaborations.

Institute of Policy and Planning Sciences, University of Tsukuba, 1-1-1 Tennodai, Tsukuba, Ibaraki 305-8573, Japan.

\section{APPENDIX}

Proof of Lemma 2 For each $i \in N$, let $\gamma_{i}: A_{-i} \rightarrow \mathbb{R}$ be such that

$$
\gamma_{i}\left(a_{-i}\right)=g_{i}(a)-G(a)
$$

for all $a_{-i} \in A_{-i}$. The function $\gamma_{i}$, found by Slade (1994) and Facchini et al. (1997), is well defined because

$$
g_{i}\left(a_{i}, a_{-i}\right)-G\left(a_{i}, a_{-i}\right)=g_{i}\left(a_{i}^{\prime}, a_{-i}\right)-G\left(a_{i}^{\prime}, a_{-i}\right)
$$

for all $a_{i}, a_{i}^{\prime} \in A_{i}$ and $a_{-i} \in A_{-i}$. Thus $g_{i}(a)=G(a)+\gamma_{i}\left(a_{-i}\right)$ for all $a \in A$. Noting 
that $\sigma_{i}\left(t_{i}\right)=\sigma_{i}^{\prime}\left(t_{i}\right)$ for all $t_{i} \in T_{i} \backslash T_{i}^{u_{i}}$, we have

$$
\begin{aligned}
& U_{i}\left(\sigma_{i}, \sigma_{-i}\right)-U_{i}\left(\sigma_{i}^{\prime}, \sigma_{-i}\right)=\sum_{t \in T} \sum_{a \in A} P(t)\left[\sigma_{i}\left(a_{i} \mid t_{i}\right)-\sigma_{i}^{\prime}\left(a_{i} \mid t_{i}\right)\right] \sigma_{-i}\left(a_{-i} \mid t_{-i}\right) u_{i}(a, t) \\
& =\sum_{\left(t_{i}, t_{-i}\right) \in T_{i}^{u_{i}} \times T_{-i}} \sum_{a \in A} P(t)\left[\sigma_{i}\left(a_{i} \mid t_{i}\right)-\sigma_{i}^{\prime}\left(a_{i} \mid t_{i}\right)\right] \sigma_{-i}\left(a_{-i} \mid t_{-i}\right) g_{i}(a) \\
& =\sum_{\left(t_{i}, t_{-i}\right) \in T_{i}^{u_{i}} \times T_{-i}} \sum_{a \in A} P(t)\left[\sigma_{i}\left(a_{i} \mid t_{i}\right)-\sigma_{i}^{\prime}\left(a_{i} \mid t_{i}\right)\right] \sigma_{-i}\left(a_{-i} \mid t_{-i}\right)\left[G(a)+\gamma_{i}\left(a_{-i}\right)\right] \\
& =\sum_{\left(t_{i}, t_{-i}\right) \in T_{i}^{u_{i}} \times T_{-i}} \sum_{a \in A} P(t)\left[\sigma_{i}\left(a_{i} \mid t_{i}\right)-\sigma_{i}^{\prime}\left(a_{i} \mid t_{i}\right)\right] \sigma_{-i}\left(a_{-i} \mid t_{-i}\right) G(a) \\
& =\sum_{t \in T} \sum_{a \in A} P(t)\left[\sigma_{i}\left(a_{i} \mid t_{i}\right)-\sigma_{i}^{\prime}\left(a_{i} \mid t_{i}\right)\right] \sigma_{-i}\left(a_{-i} \mid t_{-i}\right) G(a) \\
& =V\left(\sigma_{i}, \sigma_{-i}\right)-V\left(\sigma_{i}^{\prime}, \sigma_{-i}\right)
\end{aligned}
$$

which completes the proof.

Proof of Lemma 3 Any $\sigma_{i} \in \Sigma_{i} \backslash \Sigma_{i}^{u_{i}}$ is strictly dominated by some $\sigma_{i}^{\prime} \in \Sigma_{i}^{u_{i}}$. Thus $\sigma$ is a Bayesian Nash equilibrium if $\sigma \in \Sigma^{\mathbf{u}}$ and, for each $i \in N$,

$$
U_{i}(\sigma) \geq U_{i}\left(\sigma_{i}^{\prime}, \sigma_{-i}\right)
$$

for all $\sigma_{i}^{\prime} \in \Sigma_{i}^{u_{i}}$. Due to Lemma 2, we have

$$
U_{i}(\sigma)-U_{i}\left(\sigma_{i}^{\prime}, \sigma_{-i}\right)=V(\sigma)-V\left(\sigma_{i}^{\prime}, \sigma_{-i}\right)
$$

for all $i \in N, \sigma \in \Sigma^{\mathbf{u}}$, and $\sigma_{i}^{\prime} \in \Sigma_{i}^{u_{i}}$ because $t_{i} \in T_{i} \backslash T_{i}^{u_{i}}$ implies $\sigma_{i}\left(t_{i}\right)=\sigma_{i}^{\prime}\left(t_{i}\right)$. Therefore, any $\sigma^{*} \in \arg \max _{\sigma \in \Sigma^{u}} V(\sigma)$ is a Bayesian Nash equilibrium, if it exists.

We show that $\arg \max _{\sigma \in \Sigma^{\mathbf{u}}} V(\sigma)$ is nonempty. Let $\left\{\sigma^{k} \in \Sigma^{\mathbf{u}}\right\}_{k=1}^{\infty}$ be such that $\lim _{k \rightarrow \infty} V\left(\sigma^{k}\right)=\sup _{\sigma \in \Sigma^{\mathbf{u}}} V(\sigma)$. Consider $Q^{k} \in \Delta(T \times A)$ with $Q^{k}(t, a)=P(t) \sigma^{k}(a \mid t)$ and regard $T \times A$ as a metric space with a metric $\rho$ such that $\rho\left((t, a),\left(t^{\prime}, a^{\prime}\right)\right)=1$ if $(t, a) \neq\left(t^{\prime}, a^{\prime}\right)$ and $\rho((t, a),(t, a))=0$. Note that, for every $\varepsilon>0$, there exists a finite subset $S_{\varepsilon} \subset T$ such that $Q^{k}\left(S_{\varepsilon} \times A\right)=P\left(S_{\varepsilon}\right)>1-\varepsilon$ for all $k \geq 1$. This implies that $\left\{Q^{k}\right\}_{k=1}^{\infty}$ is tight because $S_{\varepsilon} \times A$ is compact. Thus $\left\{Q^{k}\right\}_{k=1}^{\infty}$ is relatively compact due 
to Prohorov's theorem (see Billingsley, 1968). Let $\left\{Q^{k_{l}}\right\}_{l=1}^{\infty}$ be the weakly convergent subsequence with $Q^{k_{l}} \rightarrow Q^{*}$ as $l \rightarrow \infty$.

For each $i \in N$, let $\sigma_{i}^{*} \in \Sigma_{i}^{u_{i}}$ be such that

$$
\sigma_{i}^{*}\left(a_{i} \mid t_{i}\right)=\lim _{l \rightarrow \infty} \sigma_{i}^{k_{l}}\left(a_{i} \mid t_{i}\right)
$$

for all $a_{i} \in A_{i}$ and $t_{i} \in T_{i}$. Note that the limit in the right hand side exists because

$$
\begin{aligned}
\lim _{l \rightarrow \infty} \sigma_{i}^{k_{l}}\left(a_{i} \mid t_{i}\right) & =\lim _{l \rightarrow \infty} \sigma_{i}^{k_{l}}\left(a_{i} \mid t_{i}\right) \sum_{a_{-i} \in A_{-i}} \sigma_{-i}^{k_{l}}\left(a_{-i} \mid t_{-i}\right) \\
& =\lim _{l \rightarrow \infty} \sum_{a_{-i} \in A_{-i}} Q^{k_{l}}(t, a) / P(t) \\
& =\sum_{a_{-i} \in A_{-i}} Q^{*}(t, a) / P(t)
\end{aligned}
$$

where $t_{-i} \in T_{-i}$ is such that $P(t)>0$. Then we have

$$
Q^{*}(t, a)=\lim _{l \rightarrow \infty} Q^{k_{l}}(t, a)=P(t) \lim _{l \rightarrow \infty} \sigma^{k_{l}}(a \mid t)=P(t) \sigma^{*}(a \mid t)
$$

for all $(t, a) \in T \times A$. Thus

$$
\begin{aligned}
\sup _{\sigma \in \Sigma^{u}} V(\sigma) & =\lim _{l \rightarrow \infty} V\left(\sigma^{k_{l}}\right)=\lim _{l \rightarrow \infty} \sum_{(t, a) \in T \times A} P(t) \sigma^{k_{l}}(a \mid t) G(a) \\
& =\lim _{l \rightarrow \infty} \sum_{(t, a) \in T \times A} Q^{k_{l}}(t, a) G(a)=\sum_{(t, a) \in T \times A} Q^{*}(t, a) G(a) \\
& =\sum_{(t, a) \in T \times A} P(t) \sigma^{*}(a \mid t) G(a)=V\left(\sigma^{*}\right)
\end{aligned}
$$

and $\sigma^{*} \in \arg \max _{\sigma \in \Sigma^{\mathrm{u}}} V(\sigma)$, which completes the proof.

Q.E.D. 


\section{REFERENCES}

[1] Billingsley, P. (1968): Convergence of Probability Measures. New York: John Wiley \& Sons.

[2] Blume, L. (1993): "The Statistical Mechanics of Strategic Interaction," Games and Economic Behavior, 5, 387-424.

[3] Facchini, G., F. van Megan, P. Borm, and S. Tijs (1997): "Congestion Models and Weighted Bayesian Potential Games," Theory and Decision, 42, 193-206.

[4] Hart, O., and J. Moore (1990): "Property Rights and the Nature of the Firm," Journal of Political Economy, 98, 1119-1158.

[5] Hofbauer, J., and G. Sorger (1999): "Perfect Foresight and Equilibrium Selection in Symmetric Potential Games," Journal of Economic Theory, 85, 1-23.

[6] Kajii, A., and S. Morris (1997a): "The Robustness of Equilibria to Incomplete Information," Econometrica, 65, 1283-1309.

[7] _ (1997b): "Refinements and Higher Order Beliefs: A Unified Survey," mimeo, University of Pennsylvania.

[8] Monderer, D., and L. S. Shapley (1996): "Potential Games," Games and Economic Behavior, 14, 124-143.

[9] Morris, S. (1997): "Interaction Games: A Unified Analysis of Incomplete Information, Local Interaction, and Random Matching," mimeo, University of Pennsylvania.

[10] Myerson, R. B. (1977): "Graphs and Cooperation in Games," Mathematics of Operations Research, 2, 225-229. 
[11] Myerson, R. B. (1991): Game Theory: Analysis of Conflict. Cambridge, MA: Harvard University Press.

[12] Rosenthal, R. W. (1973): "A Class of Games Possessing Pure-Strategy Nash Equilibria," International Journal of Game Theory, 2, 65-67.

[13] Slade, M. E. (1994): "What Does an Oligopoly Maximize?," Journal of Industrial Economics, 42, 45-61.

[14] Ui, T. (2000): "A Shapley Value Representation of Potential Games," Games and Economic Behavior, 31, 121-135. 


\section{NOTES}

1 I thank seminar participants at the University of Tokyo, Kyoto University, and University of Tsukuba. Financial Support from the Grant-in-Aid for Encouragement of Young Scientists is gratefully acknowledged. The usual disclaimer applies.

2 Monderer and Shapley (1996) referred to Blume (1993). See also Hofbauer and Sorger (1999).

3 Examples of potential games include the incomplete contract model of Hart and Moore (1990), the endogenous coalition formation model of Myerson $(1977,1991)$, and the congestion model of Rosenthal (1973).

$4 \bar{T}^{\mathbf{u}}=\left\{t \in T^{\mathbf{u}} \mid P(t)>0\right\}$ corresponds to $\Omega_{\mathcal{U}}$ of Kajii and Morris (1997a, Definition 2.4).

5 In Kajii and Morris (1997a), robustness of correlated equilibria is defined.

6 To the author's knowledge, we do not yet find examples of equilibria that are robust to canonical elaborations but not robust to all elaborations.

$7 \quad$ See also Morris (1997).

8 As the number of players increases, the condition of $\mathbf{p}$-dominance with $\sum_{i} p_{i}<$ 1 becomes stronger.

9 Exact potential functions of incomplete information games are discussed in Facchini et al. (1997).

10 The proof is in the Appendix.

11 The proof is in the Appendix. 